\title{
Amorphous Structure Analysis of Si Anode for Li Ion Battery
}

\author{
Yusuke Shimo $^{1}$, Akihiko Hirata ${ }^{2}$, Hisatsugu Yamasaki $^{3}$, Hiroyuki Yamaguchi $^{3}$, and Kazuaki Sato ${ }^{1}$ \\ 1. Advanced Material Engineering Division, Toyota Motor Corporation, Susono, Shizuoka, Japan. \\ 2. Advanced Institute for Materials Research, Tohoku University, Sendai, Miyagi, Japan. \\ 3. Battery Material Engineering and Research Division, Toyota Motor Corporation, Susono, Shizuoka, \\ Japan.
}

Lithium-ion batteries (LIBs) are major energy storage in portable electronic devices, hybrid/plug-in hybrid vehicles (HV/PHV), and electric vehicles (EV). Especially, with regard to the use in HV/PHV and EV, LIBs are expected to have much higher gravimetric and volumetric energy densities than current LIBs. Silicon is an attractive material as an alternative anode of LIB because its theoretical capacity is more than tenfold greater than the current carbon (graphite) anode, so LIBs containing Si anode have been the subject of much recent investigation [1]. However, Si has some problems to be used as LIB anode. One of the problems is "hysteresis"; cell voltage (Open Circuit Voltage; OCV) profiles at the discharging state decrease compared with the charging state. Although hysteresis leads to declining the performance of LIB, the reason why it occurs has been poorly understood. Recently, a study was conducted on macroscopic analysis of voltage hysteresis and it was reported that the mechanical stress of Si anode might have relation to hysteresis [2]. However, no study has been reported on the microscopic analysis to investigate hysteresis of Si anode LIB. Therefore, in this work, we aimed to study the microscopic mechanism of hysteresis.

It is known that $\mathrm{Si}$ becomes $\mathrm{Li}_{\mathrm{x}} \mathrm{Si}$ alloy during electrochemical $\mathrm{Li}$ insertion and its structure changes from crystalline to amorphous-crystalline mixture at sub-micrometer scale [3] (Figure 1 shows TEM image of amorphous $\mathrm{Li}_{\mathrm{x}} \mathrm{Si}$ ). Therefore, we built hypothesis that the structure of amorphous $\mathrm{Li}_{\mathrm{x}} \mathrm{Si}$ might have relation to the hysteresis and challenged to analyze it. To analyze the amorphous structure, we applied STEM diffraction using sub-nanometer electron beam (Angstrom Beam Electron Diffraction; ABED) [4]. Since it is difficult to acquire isolated diffraction patterns from amorphous using conventional TEM equipment, we used a Cs-corrected STEM (JEOL ARM-200F operated at 200keV) equipped with a cold-FEG and a special-designed condenser aperture (hole diameter; 3.5um). Furthermore, LIB materials are so sensitive to air that we applied air-isolated (vacuum or Ar atmosphere) process from sample preparation to ABED experiment.

Figure 2 shows a diffraction pattern acquired from amorphous $\mathrm{Li}_{\mathrm{x}} \mathrm{Si}$. More than $5000 \mathrm{ABED}$ patterns were acquired in total and indicated that the discharging state tended to have patterns at low scattering angle compared with the charging state. In addition, as a result of structure identification using simulated ABED patterns, it was suggested that amorphous LixSi at the discharging state tended to have longer Si-Si bond than the charging state. Consequently, there is a possibility that the structural difference between charging and discharging state causes hysteresis. 


\section{References:}

[1] U. Kasavajjula, et al, J. Power Sources 163 (2007), p. 1003.

[2] B. Lu, et al, Phys. Chem. Chem. Phys. 18 (2016), p. 4721.

[3] P. Limthongkul, et al, J. Power Sources 119-121 (2003), p. 624.

[4] A. Hirata, et al, Nat. Commun. 7 (2016), p. 11591.
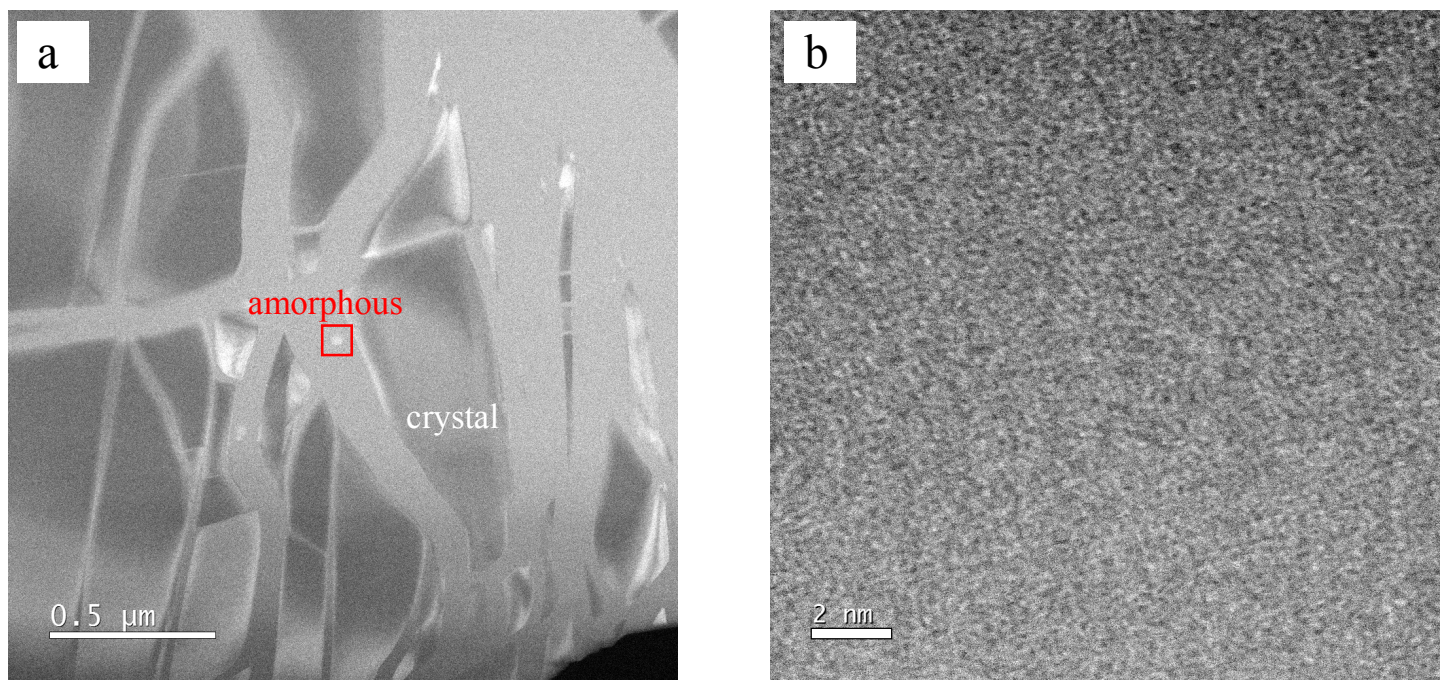

Figure 1. (a) ADF-STEM Images of Si anode at discharging state. (b) High resolution STEM image of amorphous $\mathrm{Li}_{\mathrm{x}} \mathrm{Si}$.
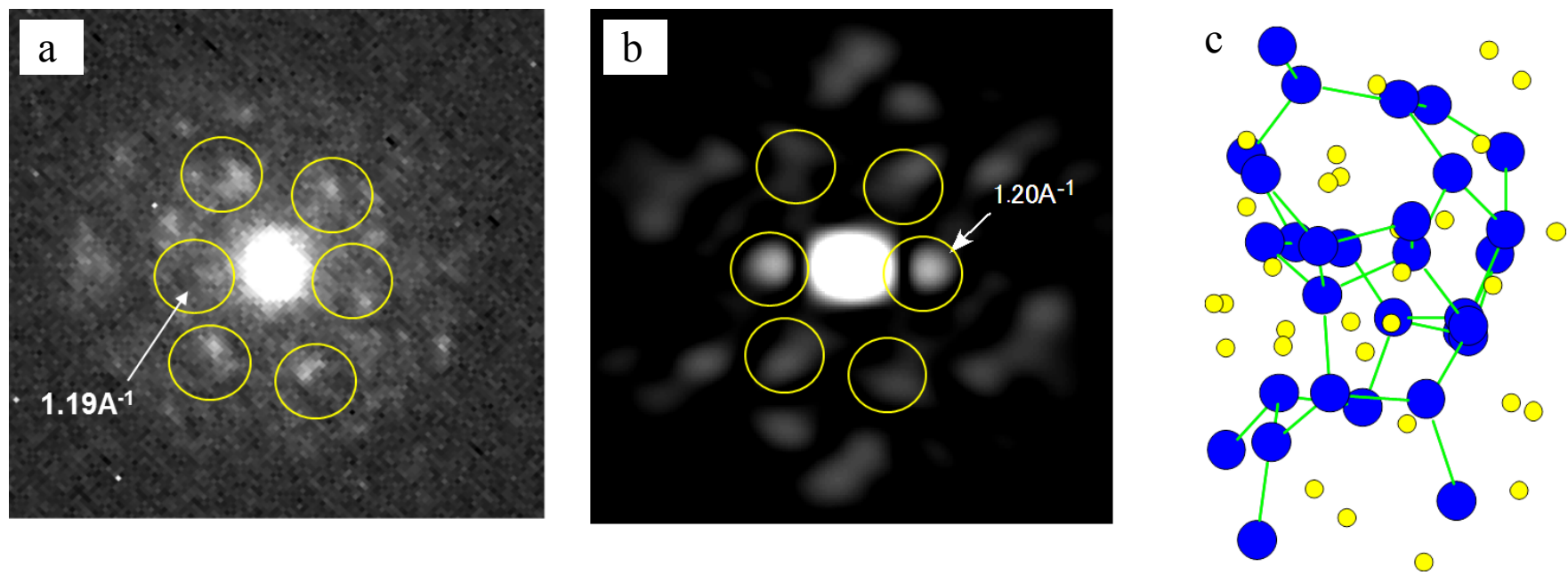

Figure 2. (a) Angstrom beam electron diffraction (ABED) pattern obtained amorphous $\mathrm{Li}_{\mathrm{x}} \mathrm{Si}$. (b) Simulated diffraction pattern from LiSi in (c). 